\title{
Insight into the Microcosm of the Human Growth Hormone and its Interactions with Polymers and Copolymers: A Molecular Dynamics Perspective
}

Zahra Lotfi-Sousefi ${ }^{1}$, Faramarz Mehrnejad ${ }^{1 *}$, Somayeh Khanmohammadi ${ }^{1}$, S. Fatemeh Kaboli ${ }^{1}$

${ }^{1}$ Department of Life Science Engineering, Faculty of New Sciences and Technologies, University of Tehran, 14395-

1561, Tehran, Iran

*.Corresponding author: mehrnejad@ut.ac.ir 

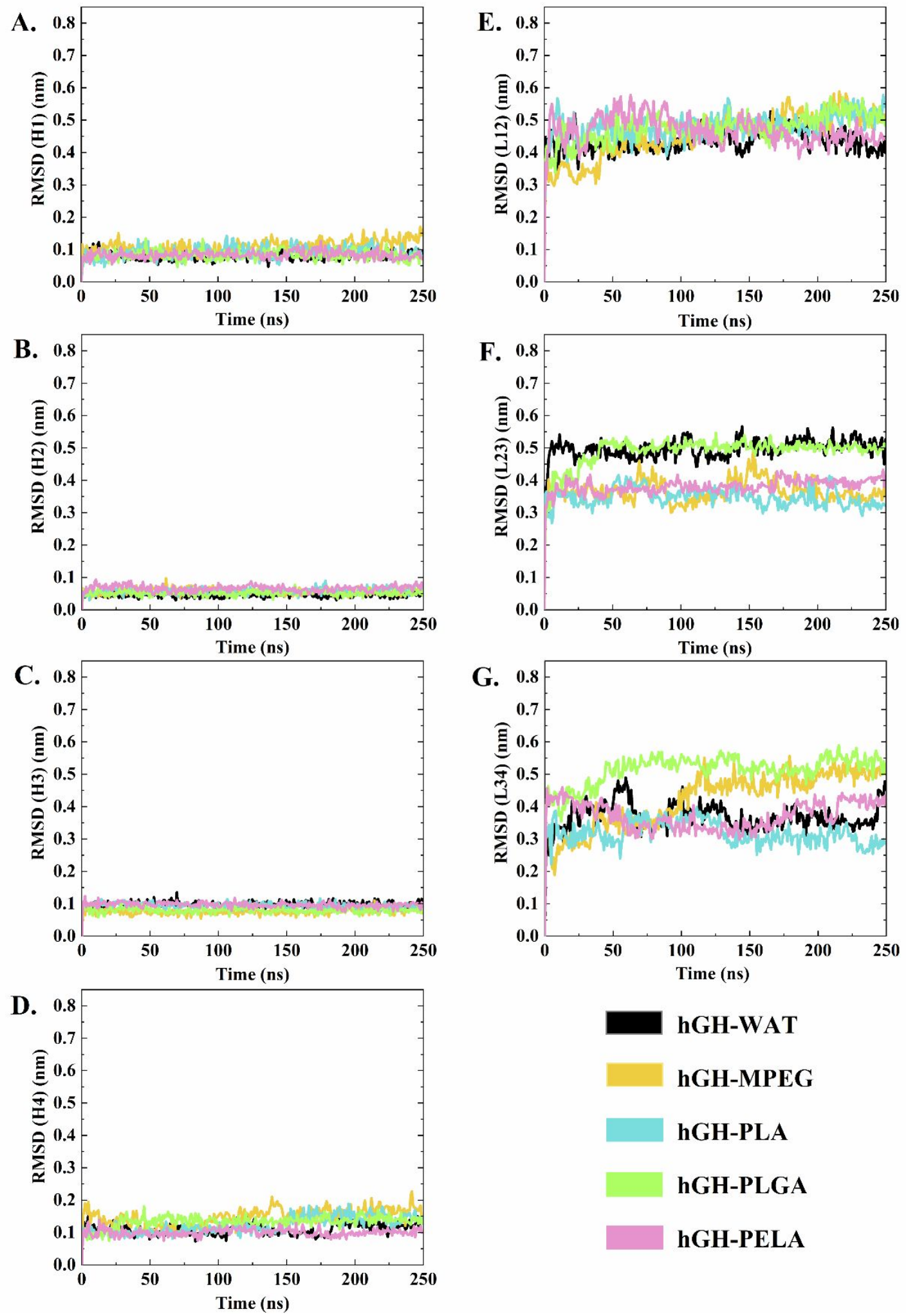

Figure S1. The C-alpha RMSD plot for four $\alpha$-helices: (A) H1, (B) H2, (C) H3, (D) H4, and three loops connecting helices: (E) L12, (F) L23, (G) L34. 


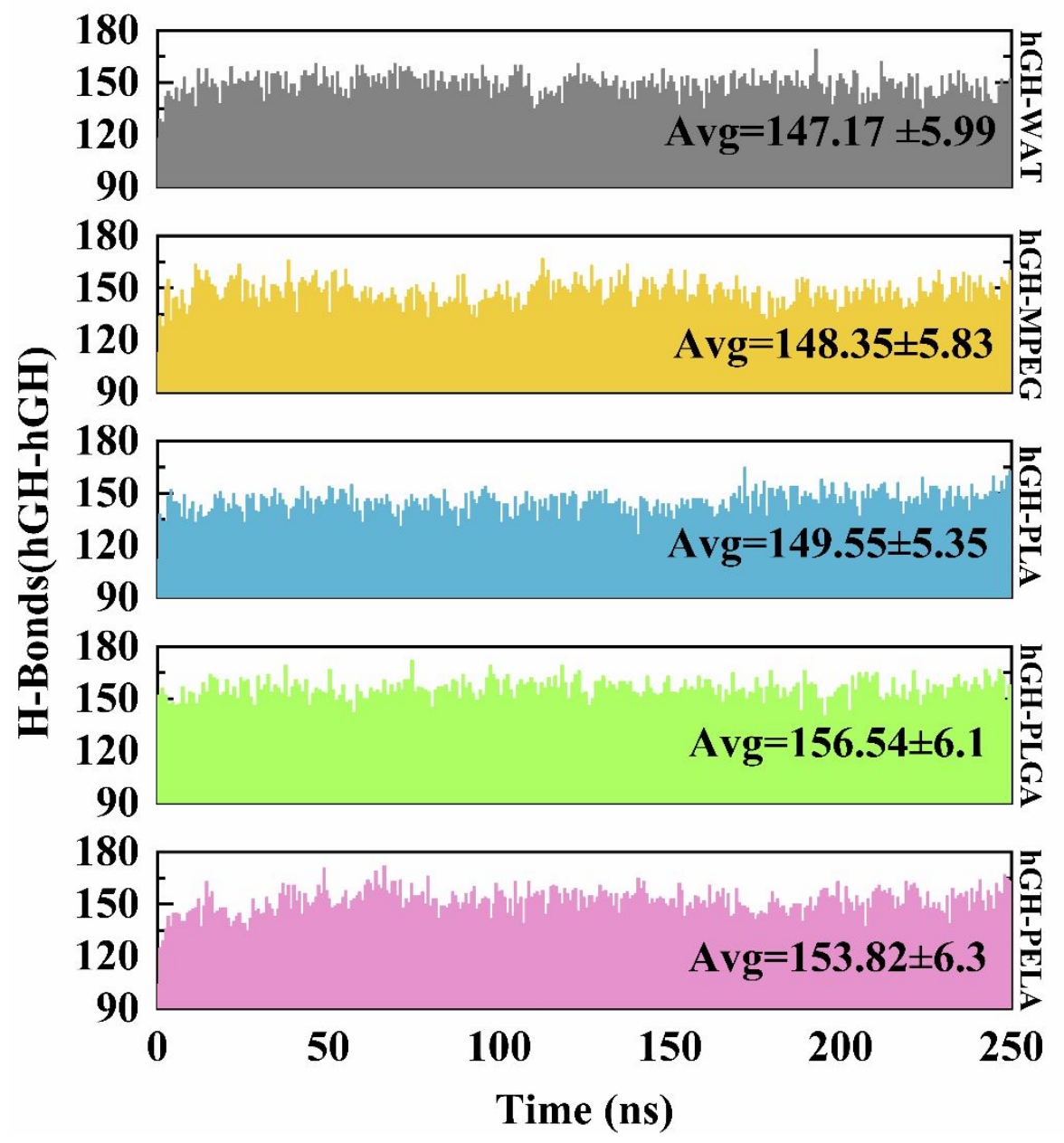

Figure S2. The number of intramolecular H-bonds over 250 ns. 

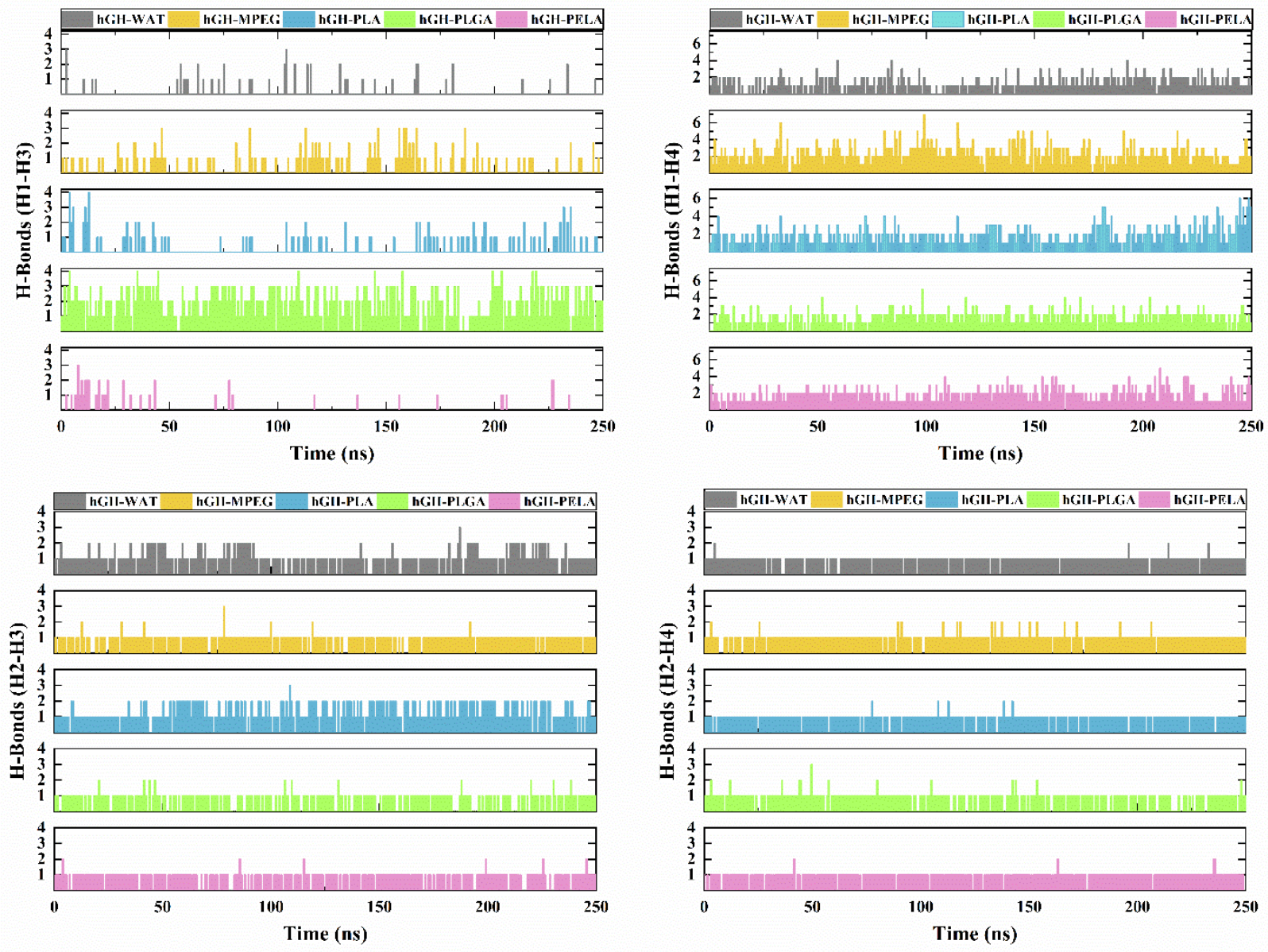

Figure S3. The number of interhelical H-bonds over 250 ns. The number of H-bonds between (A) H1-H3, (B) H2-H3, (C) H1-H4, (D) H2-H4. No H-bond is formed between other helices. 

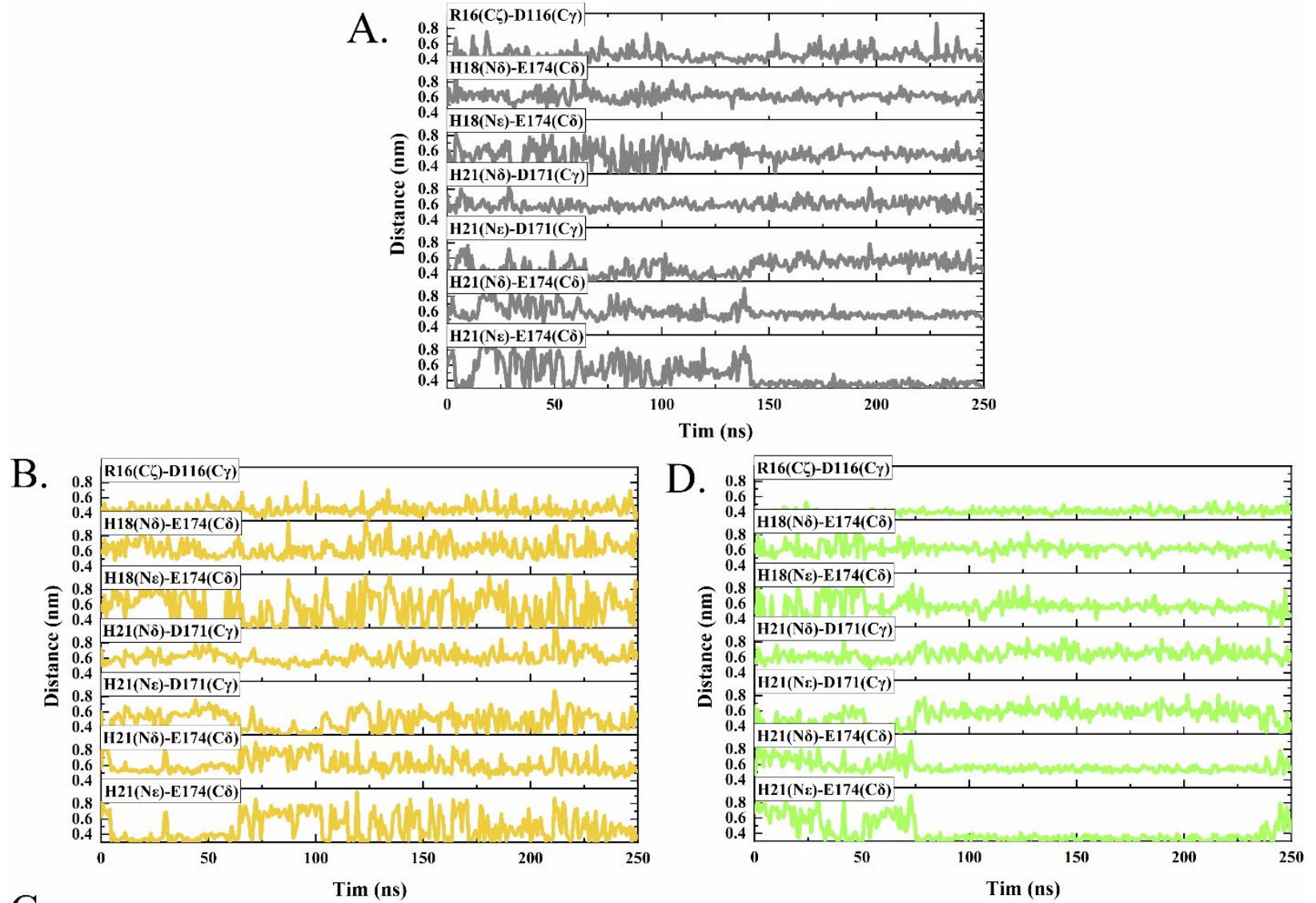

C.

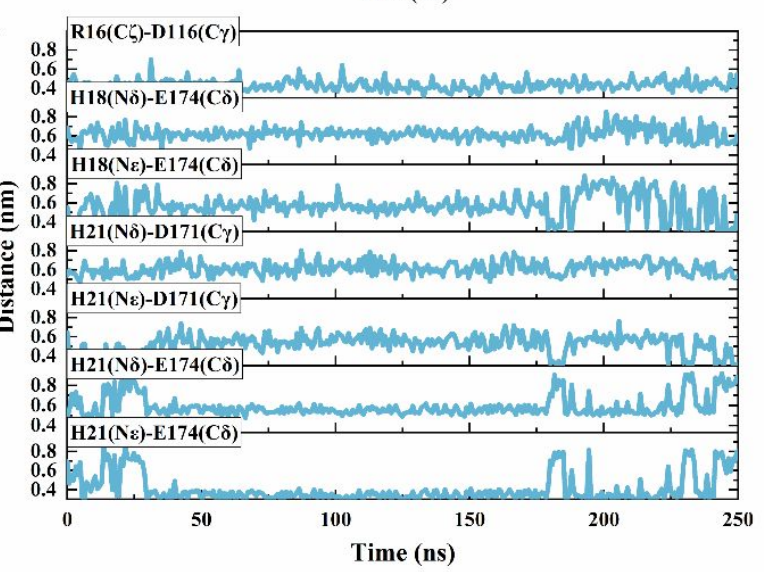

E.

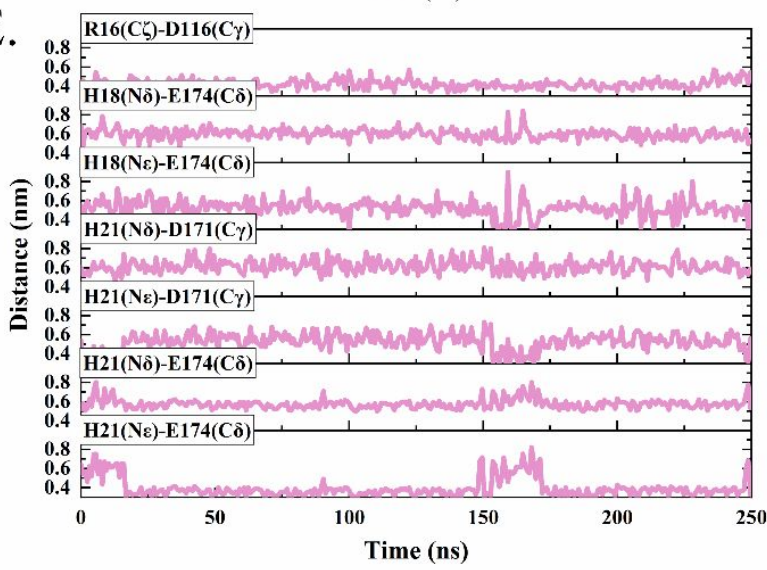

Figure S4. The Distance between residues involved in interhelical salt bridge formation in hGH, during the 250 ns MD simulations. 